\title{
Atenção primária à saúde: diretrizes, desafios e recomendações. Revisão de bibliografia internacional
}

I ${ }^{1}$ Luiz Gustavo Silva Souza, ${ }^{2}$ Maria Cristina Smith Menandro I

Resumo: Apresentamos uma revisão de bibliografia internacional que teve por objetivo identificar e analisar a diversidade de temas associados à Atenção Primária à Saúde, especialmente o cotidiano de trabalho de seus profissionais, suas diretrizes e seus desafios políticos e clínicos. Analisamos os resumos de 105 artigos publicados entre 1999 e 2009 . Selecionamos, para análise, 34 artigos identificados de acordo com critérios específicos. Criamos categorias temáticas e comparamos os dados com certa literatura brasileira sobre o tema. Os artigos centram-se no profissional de medicina e enfatizam a noção de medicina baseada em evidências. Abordam principalmente a implementação de diretrizes na Atenção Primária e as relações entre profissionais e usuários. Admitem a complexidade da Atenção Primária e a importância de aproximação à realidade dos pacientes. Entretanto, não abordam a intersetorialidade, não questionam radicalmente o especialismo curativista e não mencionam a promoção de cidadania, autonomia e cogestão junto aos usuários. Falam da necessidade de abordar problemas "humanos" nos níveis de implementação de diretrizes, encontro clínico etc., mas se mostram distanciados das ciências humanas.

> Palavras-chave: atenção primária à saúde, profissionais de saúde, diretrizes para o planejamento em saúde, medicina baseada em evidências.
1 Doutorando do Programa de Pós-Graduação em Psicologia da Universidade Federal do Espírito Santo (PPGPUFES). Endereço eletrônico: luizsouza@hotmail.com

2 Professora Doutora do PPGP-UFES. 


\section{Introdução}

Há pouco mais de trinta anos, a Declaração de Alma-Ata, publicada pela Organização Mundial da Saúde (1978), conclamou a comunidade internacional a atingir o objetivo de "saúde para todos", priorizando os sistemas nacionais de atenção primária. Em muitos países, fortaleceu-se a ideia de que os modelos de atenção à saúde centrados no hospital são insuficientes para atingir bons resultados. As três últimas décadas foram testemunhas de diferentes esforços de reorganização dos sistemas sanitários nos países ditos "ocidentais", todos eles dando grande importância à atenção primária à saúde (COELHO, 2008).

Apesar da grande relevância do tema, nota-se que a produção acadêmica brasileira sobre ele ainda é escassa e dispersa, estabelecendo pouco diálogo com a literatura internacional (BAPTISTA; FAUSTO; CUNHA, 2009). Com este artigo de revisão, procuramos oferecer uma contribuição para a realização desse diálogo. Buscamos verificar como a atenção primária à saúde (APS) foi abordada em periódicos científicos internacionais na última década (1999 a 2009), comparando os resultados com certa literatura brasileira sobre o tema (CAMPOS, 2003; CAMPOS et al., 2008; FRANCO; MERHY, 2007; COELHO, 2008; SOUSA; HAMANN, 2009).

$\mathrm{Na}$ revisão de literatura nacional efetuada por Baptista, Fausto e Cunha (2009), o trabalho de Giovanella (2006) foi a única referência brasileira sobre APS em contexto internacional. Giovanella (2006) descreve reformas na APS europeia e argumenta que o conhecimento a respeito dessas transformações pode ajudar a pensar o caso brasileiro. A autora centrou sua análise na organização geral dos sistemas de saúde, nos modos de financiamento e no papel desempenhado pela APS em relação aos serviços especializados.

Com o presente trabalho, nosso intuito também será contribuir para localizar as reflexões e pesquisas sobre APS no Brasil no quadro da produção científica internacional. $\mathrm{O}$ objetivo de nossa revisão bibliográfica foi identificar e analisar a diversidade de temas associados à APS, especialmente no que se refere ao cotidiano de trabalho de seus profissionais e aos seus desafios políticos e clínicos. Perguntamo-nos quais as características gerais dessa produção científica (localização geográfica, métodos de análise, etc.), quais recomendações, potencialidades e dificuldades são associadas à APS de forma recorrente. 
Realizamos uma revisão bibliográfica por meio do Portal de Periódicos da CAPES. Escolhemos duas editoras que publicam, cada uma, diversas revistas internacionais: Biomed Central e Sage. Justificaremos essas escolhas a seguir. A Biblioteca Virtual em Saúde (2007) definiu a Biomed Central como um dos maiores divulgadores mundiais de pesquisa científica com acesso aberto. Por serem de livre acesso, podemos inferir que seus artigos tenham boas possibilidades de penetração e de impacto. Os periódicos publicados por essa editora abordam temas mais especificamente biomédicos. A escolha da editora Sage foi feita para contrabalancear essa tendência, já que, segundo o Portal de Periódicos, a Sage enfoca especialmente as ciências humanas e sociais aplicadas.

Utilizamos a opção de "pesquisa avançada" nos endereços eletrônicos das duas editoras. A palavra-chave escolhida foi "primary care" (atenção primária) que deveria aparecer como "exact phrase" (expressão inteira) nos títulos dos artigos. Partimos do critério de que a presença dessa expressão no título era o sinal necessário de uma abordagem direta do tema. Restringimos o período à última década (1999 a 2009). Com essa configuração de pesquisa, obtivemos resposta de 311 artigos na Biomed Central e de 167 artigos na Sage, um total de 478 artigos.

Em seguida, lemos os títulos dos 478 artigos e aplicamos a eles os seguintes critérios de seleção: a) foram excluídos aqueles que abordavam alguma doença ou transtorno em específico (por exemplo, diabetes, hipertensão, depressão, etc.); b) foram excluídos os artigos que se restringiam a um grupo determinado de pacientes (crianças, idosos, doentes terminais, etc.) e também aqueles que tratavam especificamente da informática na APS. Aplicando esses critérios, chegamos a 105 artigos.

Lemos o resumo desses 105 trabalhos, excluindo cinco "protocolos de estudo" (projetos ainda não executados quando da publicação) e classificando-os em quatro categorias: cotidiano de trabalho $(n=34)$; pacientes da APS $(n=18)$; formas de pesquisa na APS $(n=14)$ e política e gestão da APS $(n=34)$. Para o presente relato, escolhemos explorar somente a primeira categoria, pois estamos interessados sobretudo nos temas e desafios que caracterizam o cotidiano de trabalho dos profissionais.

Portanto, lemos integralmente 34 artigos. Realizamos uma caracterização geral desses estudos, sistematizando dados objetivos como país de origem, 
métodos, etc. Em seguida, dividimos os estudos em subcategorias temáticas e os analisamos de forma descritiva, buscando responder às seguintes questôes: quais são os principais resultados desses estudos? Quais são os principais obstáculos, identificados pelos autores, para uma boa atenção primária à saúde? Que princípios e/ou práticas esses mesmos autores recomendam?

\section{Resultados e análises}

\section{Caracterização geral dos artigos}

Todos os artigos analisados $(\mathrm{n}=34)$ foram publicados em inglês. Eles foram classificados em: relatos de pesquisa $(n=26)$, estudos teóricos $(n=4)$, revisões de literatura $(n=2)$ e relatos de implementação/intervenção $(n=2)$. Como afirmamos acima, a principal característica do conjunto de estudos que analisamos foi a abordagem direta dos profissionais da APS e de seu cotidiano de trabalho. Em decorrência disso, quase todos os relatos de pesquisa ( 25 em 26) escolheram uma ou mais categorias profissionais como sujeitos de pesquisa, ou seja, como informantes. Apenas um deles abordou os profissionais de forma indireta (avaliando pacientes).

Os médicos foram priorizados como informantes, sendo incluídos em 23 relatos $(88,4 \%)$, dentre os quais apareceram exclusivamente em 14 (53,8\%). A segunda categoria profissional mais citada, a dos enfermeiros, foi eleita como sujeito de pesquisa em apenas nove estudos (34,6\%) e exclusivamente somente em um $(3,8 \%)$. É interessante observar que apenas uma pesquisa abordou psicólogos, por exemplo. Ou seja, na bibliografia analisada, pesquisar o cotidiano da APS equivaleu a pesquisar os médicos e, secundariamente, os enfermeiros. Esse dado talvez reflita uma diferença existente entre o sistema de saúde brasileiro (organizado em torno da Estratégia ou "Programa" Saúde da Família - PSF) e os sistemas de outros países. Sousa e Hamann (2009) afirmam esse ponto como parte da originalidade do sistema brasileiro: "diferentemente da medicina familiar vigente em vários países da Europa, no Canadá e na Oceania, o PSF pressupóe o trabalho multiprofissional e em equipe como processo básico para a integralidade do cuidado na atenção primária à saúde" (SOUZA; HAMANN, 2009, p. 1.328).

Os relatos de pesquisa e revisões de literatura $(n=28)$ foram classificados em "quantitativos" e "qualitativos". Sabemos que se trata de categorias cuja diferenciação é complexa (GUNTHER, 2006), por isso precisamos explicitar claramente o que estamos chamando de quantitativo e qualitativo. Aplicamos essa classificação aos 
estudos segundo o método de análise utilizado. Nosso critério foi o seguinte: se o estudo recorreu a instrumentos estatísticos (análises de variância, etc.) para analisar seus dados e responder à sua questão central, então ele foi nomeado quantitativo. Se o método de análise incluiu procedimentos assemelhados à análise de conteúdo temática, o estudo foi chamado de qualitativo. Verificamos quase o mesmo número de estudos quantitativos $(\mathrm{n}=13)$ e qualitativos $(\mathrm{n}=14)$ e apenas um estudo "misto" (este último com análise quantitativa de filmagens de consultas e análise qualitativa de entrevistas em profundidade com pacientes).

Nas pesquisas caracterizadas como quantitativas, os métodos de coleta de dados mais utilizados foram: questionários e escalas $(\mathrm{n}=11)$ e registros em prontuário $(\mathrm{n}=2)$. Nos qualitativos, foram: entrevistas e entrevistas semiestruturadas $(n=8)$ e grupos focais $(n=5)$. Quanto ao ano de publicação, os anos com maior frequência foram 2007 e 2008, com sete e nove artigos respectivamente, o que talvez indique um interesse crescente por esse tipo de estudo a partir de meados da década analisada (o ano de 2009 foi considerado somente até agosto).

Quanto à distribuição geográfica dos artigos, verificamos que a grande maioria teve origem na América do Norte ( $\mathrm{n}=22 ; 64,7 \%)$, seguida por Europa $(\mathrm{n}=11) \mathrm{e}$ Ásia $(\mathrm{n}=1)$. Os artigos dos Estados Unidos predominaram $(\mathrm{n}=17)$ e houve cinco trabalhos canadenses. Dentre os europeus, somente Grã-Bretanha $(n=6)$, Suécia $(\mathrm{n}=3)$ e Espanha $(\mathrm{n}=2)$ estiveram representados. O artigo asiático é israelense. Chama a atenção a total ausência de América do Sul, África e Oceania, bem como de outros países europeus e asiáticos expressivos, como França, Alemanha, Rússia, China, Índia e Japão. Percebe-se que não há qualquer artigo proveniente dos chamados "países em desenvolvimento".

Portanto, o discurso sobre a APS que analisamos aqui é aquele dos chamados países "desenvolvidos", principalmente anglófonos. É possível que isso tenha acontecido em parte por causa das editoras escolhidas para a pesquisa. De qualquer maneira, esse dado talvez sugira que os acadêmicos dos "países em desenvolvimento" estão privilegiando outros veículos e formas de publicação.

Cerca de um terço dos artigos $(n=11)$ citou diretamente a noção de "medicina baseada em evidências" (MBE). Lopes (2000) define a MBE como um movimento relacionado ao ensino e à prática da medicina. $\mathrm{O}$ objetivo da $\mathrm{MBE}$ seria melhorar a prática médica, integrando a clínica ao uso racional e criterioso das melhores evidências científicas disponíveis. Tal movimento recebe adesões, mas também 
críticas. Sayd e Moreira (2000), por exemplo, a partir de uma análise histórica e filosófica do ceticismo em medicina, negam-lhe o estatuto de "novo paradigma".

Como veremos no tópico seguinte, muitos estudos tomaram como objeto experiências, conhecimentos, percepções, crenças, atitudes, comportamentos e práticas de profissionais de saúde. Como se sabe, esses objetos são temas consagrados nos campos da sociologia, da psicologia e da psicologia social. Era de se esperar que os estudos analisados fizessem uso direto de teorias desses campos de conhecimento para tratar seus dados. Entretanto, não foi isso que verificamos. Apenas três estudos utilizaram efetivamente teorias para analisar os resultados obtidos: Giddens' Structuration Theory (GENEAU et al., 2008); Cognitive Continuum Theory (RANDELL et al., 2009) e Diffusion of Innovation Theory (BEAULIEU et al., 2008). Retomaremos esse ponto na discussão.

\section{Análise descritiva dos temas dos artigos}

Os artigos analisados foram classificados em três categorias temáticas, que foram nomeadas como se segue: a) Incorporação de diretrizes e conhecimentos na APS; b) Relações entre profissionais e pacientes; c) Avaliação de programas e intervenções. Essa classificação foi feita de forma mutuamente excludente. Entretanto, é importante dizer que a inclusão em uma categoria significa ênfase em determinado tema (e não sua abordagem exclusiva). A seguir, passamos a descrever cada um desses temas ordenados de "a" a "c".

\section{a) Incorporação de diretrizes e conhecimentos na APS}

Mais da metade dos estudos que analisamos $(\mathrm{n}=18)$ destacou a incorporação de diretrizes e conhecimentos à prática cotidiana de profissionais ou serviços de APS. Dentre esses estudos, identificamos referências a três tipos de diretrizes: científicas (chamadas de "evidências"), clínico-políticas (derivadas de recomendações de órgãos gestores nacionais e internacionais) e administrativas (referentes a modificações gerais dos sistemas de saúde e a características dos ambientes organizacionais).

Os estudos que enfocaram a incorporação de diretrizes científicas na APS deram destaque à noção de "medicina baseada em evidências" (MBE). Tiveram como objetivos: pesquisar sentidos atribuídos por médicos à noção de "evidência"; fatores que levam médicos a tomar decisões "contrárias às evidências”; percepçôes 
sobre os efeitos das diretrizes nas práticas; modelos e intervenções para implementação de práticas baseadas em evidências.

Alguns autores se mostraram entusiastas da $\mathrm{MBE}$, recomendando expressamente sua adoção na APS (NEMETH et al., 2008; FEIFER et al., 2006), apesar das controvérsias. Entretanto, mesmo autores favoráveis à MBE (NEMETH et al., 2008) partiram do pressuposto de que os serviços têm grandes dificuldades de implementá-la; de que a qualidade da APS é muito variável; de que há pouco uso, mal uso ou abuso de diretrizes clínicas e de que a atenção primária à saúde ocorre em sistemas complexos, sem rotina clara, com demandas que competem entre si e com profissionais muitas vezes inertes (desmotivados e com rotinas estereotipadas). Essas preocupações ecoam nos estudos de autores que se mostraram mais cautelosos com a MBE (BEAULIEU et al., 2008; TRACY et al., 2003; GUERRA et al., 2008).

Há uma preocupação crescente de que princípios fundamentais da APS (atenção focada no cliente, por exemplo) estejam em contradição irremediável com princípios da MBE. Médicos apresentaram atitudes favoráveis à MBE, mas frequentemente as expectativas dos pacientes e as características dos serviços de saúde os levaram a tomar decisões contrárias às evidências. Recomenda-se então a adoção de modelos de MBE atentos a esses fatores "externos" (TRACY et al., 2003). O estudo de Beaulieu et al. (2008) ressaltou outro problema: os pesquisadores e profissionais pesquisados adotaram diferentes noções de "evidência" (advindas justamente dos fatores contextuais percebidos e tomados em conta pelos profissionais). Guerra et al. (2008) constataram que o impacto das diretrizes para o comportamento clínico específico que pesquisaram era pequeno. Esses autores evocaram a hipótese de que médicos estejam desenvolvendo uma espécie de "fadiga de diretrizes" ("guideline fatigue"), já que as recebem em grande quantidade e de um número também elevado de organizações gestoras.

Outro conjunto de estudos abordou a incorporação de diretrizes que nomeamos "clínico-políticas". Trata-se de recomendações expressas por políticas e organizações de saúde nacionais e internacionais, relacionadas aos papéis da APS e que têm reflexo na prática clínica. Alguns artigos elegeram temas específicos, como prevenção, promoção de estilo de vida saudável, holismo, visitas domiciliares, comunicação com equipes de apoio consultivo e "medicina integrativa". Outros estudos trataram da incorporação desse tipo de diretrizes em âmbito mais geral. 
Dois artigos se dedicaram diretamente ao tema da prevenção na APS. Pollak et al. (2008) mostraram que o tempo despendido por médicos com a atividade de educação para prevenção é em geral insuficiente. Partiram do arcabouço da MBE e verificaram que as evidências disponíveis não parecem orientar a educação para prevenção. Mirand et al. (2003) estudaram percepções e crenças de médicos sobre práticas preventivas, buscando entender por que elas são preteridas. Mostraram que os participantes percebem "o médico" como "profissional que faz o diagnóstico e que provê a solução imediata para o problema", ao mesmo tempo em que entendem o paciente como alguém que quer essa solução imediata. Os médicos pesquisados se definiram como "líderes da terapêutica" e como pouco capazes de trabalhar em equipe. Eles concordaram com a ideia de que a APS é a principal responsável pela prevenção. Entretanto, apresentaram crenças de que conversar sobre prevenção não é papel do médico (desperdiça seu tempo) e de que mudar a mentalidade dos pacientes em direção à corresponsabilização sobre seu estado de saúde é muito difícil. Os médicos relataram não ter treinamento para prevenção primária na graduação.

Mirand et al. (2003) insistiram na importância de maior atenção à prevenção por parte dos médicos, enquanto Pollak et al. (2008) sugeriram dividir essa tarefa com outros profissionais (citando a crescente falta de médicos generalistas) e que as diretrizes para prevenção apresentadas aos médicos sejam simplificadas e padronizadas.

O tema "estilo de vida saudável", trabalhado por Grandes et al. (2008), inclui promoção de dieta balanceada, exercício físico, etc. Os profissionais pesquisados consideraram possível e necessário integrar atividades de promoção de estilo de vida saudável à APS, mas mostraram acreditar que ajudar as pessoas a mudar seu estilo de vida é difícil; que modelos de intervençôes eficazes estão disponíveis, mas que eles não se encaixam no funcionamento atual da APS (centrada na "cura de doenças") e que faltam intervenções para tratar combinações de risco. A falta de tempo é um dos principais obstáculos, há sobrecarga de trabalho, dificuldades em mudar a prática clínica e falta de uma "abordagem sócio-ecológica".

Esses problemas também compareceram na discussão da noção de holismo apresentada por Strandberg et al. (2007). Os autores preconizaram que a clínica da APS se baseie em uma visão holística, ou seja, que considere não só a doença, mas sim o paciente em sua complexidade psicossocial (aproximando-se 
do conceito de clínica ampliada proposto, no Brasil, por CAMPOS, 2003). Os profissionais pesquisados definiram a visão holística como aquela que considera a pessoa como um todo e falaram da necessidade de experiência e de treinamento específico para construir essa atitude. No estudo de Sundberg et al. (2007), além da abertura para uma visão mais completa dos pacientes, frisou-se a importância de apostar em alternativas terapêuticas (diferentes da clínica médica tradicional). Os médicos e gestores entrevistados pelos autores afirmaram que a APS deveria incluir terapias complementares que sejam de eficácia comprovada, somando-as aos tratamentos convencionais ("medicina integrativa").

A única pesquisa-intervenção da amostra (LEONARDO; CHECCO; COGGINS, 2001) enfocou a atuação de enfermeiros na APS de uma região rural, descrevendo a população dessa região, e mostrando relações entre as principais doenças e o estilo de vida dessa população, o que permitiu formular diretrizes clínico-políticas para o sistema de saúde local. Enfatizou-se que os serviços de APS devem sempre centrar-se nas necessidades e peculiaridades das comunidades, muitas vezes “multiculturais". Rinke e Holt (2000) também partiram da ideia de aproximação da comunidade, para frisar a necessidade das visitas domiciliares. Os autores argumentaram que o atendimento domiciliar deveria ser o centro do sistema de saúde. Ele permitiria fornecer atendimento aos que dele estão alijados, prevenir internações e promover colaboração entre médicos e enfermeiros.

A colaboração entre profissionais também foi tema de Dobscha et al. (2007), que pesquisaram as maneiras preferenciais de comunicação entre equipes de saúde básica e equipes de apoio consultivo. Os profissionais pesquisados pelos autores mostraram preferir contatos "breves" com essas equipes (obtendo informaçōes resumidas dos pacientes por telefone ou e-mail, com pouca ênfase em encontros presenciais).

Hsiao e Boult (2008) revisaram artigos sobre a relação entre qualidade (técnica e "humana") do atendimento na APS e índices de resultados. Verificaram que faltam estudos mais conclusivos para mostrar que uma melhor relação médicopaciente (com maior comunicação, abordagem holística e comunitária) realmente leva a melhores índices de resultados. Entretanto, esse ceticismo não pareceu ser compartilhado pelo conjunto dos estudos que analisamos, que muitas vezes tomaram a dita associação como pressuposto.

Finalmente, outro conjunto de estudos abordou a incorporação de diretrizes que chamamos de "administrativas", enfocando efeitos da gestão e do ambiente 
organizacional dos serviços no cotidiano de trabalho. Em dois estudos canadenses, os pesquisadores se interessaram em analisar visóes dos médicos sobre uma reforma da APS (HUNTER et al., 2004) e em investigar como os determinantes organizacionais influenciam o trabalho do médico e suas tomadas de decisão (GENEAU et al., 2008). Duas outras pesquisas abordaram o tema da satisfação dos profissionais com seu trabalho e sua relação com os modos de gestão (MARTINFERNANDEZ et al., 2007; FREEBORN; HOOKER; POPE, 2002).

Os médicos pesquisados por Hunter et al. (2004) não esperavam aderir a uma reforma do sistema de APS. Desaprovavam o método de pagamento e de agendamento (centralizado) de pacientes, alegando perda de autonomia. Aprovavam as propostas de atendimento noturno e de financiamento especial para atividades preventivas. O maior fator preditor para "aderir à reforma" foi "concordar com novo método de pagamento". Os resultados levaram os autores a preconizar a participação dos médicos na formulação das políticas gerenciais e sugerem que a questão salarial é central para essa categoria. Em consonância, Geneau et al. (2008) verificaram que a forma de pagamento é um dos principais fatores que influenciam os médicos e suas decisões clínicas, ao lado de outros, como interação com a equipe, exigências dos pacientes e existência de outros serviços de saúde na localidade. Os autores perceberam que pressões organizacionais nocivas levam a menores tempos de consulta, mais pedidos de exames, menos comunicação médico-paciente, menos atenção ao aconselhamento e à prevenção e maior ansiedade dos médicos, podendo levar a uma naturalização prejudicial da prática médica (cristalização da rotina).

Martin-Fernandez et al. (2007) perceberam correlações entre uma reforma que implicou descentralização administrativa e a percepção de qualidade de vida por parte de profissionais. Os resultados a que chegaram apoiam a sugestão de promover a participação dos profissionais na formulação da política e da gestão. Freeborn, Hooker e Pope (2002) pesquisaram médicos, enfermeiros e auxiliares e mostraram que o alto número de pacientes e o curto tempo disponível para eles eram os principais fatores de insatisfação profissional. Verificaram que enfermeiros e auxiliares são mais insatisfeitos e sugeriram que o sistema lhes permitisse maior controle sobre seu trabalho e melhor remuneração.

May et al. (2007) aplicaram um modelo de análise a dois ensaios clínicos e afirmaram que a implementação de procedimentos na APS (contexto 
complexo) tem que considerar fatores sócio-interacionais: a organização do serviço, a prática dos profissionais e sua interação com os pacientes. Os autores defendem a validade dos ensaios clínicos como metodologia científica, mas consideram que as noções e os métodos das ciências sociais devem estar intrinsecamente incorporados à montagem desses ensaios. Voltaremos mais adiante a essa aproximação com as ciências sociais.

\section{b) Relaçôes entre profissionais e pacientes}

Doze artigos centraram foco sobre as relações entre profissionais e pacientes. Todos eles deram ênfase às relaçôes "de consultório", em um corte temporal localizado (três deles, gravando consultas). Não houve estudo que se dedicasse a pesquisar de forma longitudinal a relação entre determinados profissionais e pacientes, em uma comunidade.

Dentre esses artigos, quatro analisaram relações estabelecidas com pacientes que possuem características específicas: baixa renda, minorias étnicas (CARDARELLI; CHIAPA, 2007; CHAUDRY et al., 2003), homens e mulheres negros (DIMATTEO; MURRAY; WILLIAMS, 2009) e pessoas com deficiência mental (JONES et al., 2008). Esses estudos enfatizaram a necessidade de comunicação profissional-paciente e as dificuldades enfrentadas por esses grupos específicos, (que apresentam níveis mais altos de morbidade) para ter acesso à APS de qualidade.

Chaudry et al. (2003) descreveram algumas dessas dificuldades. Do ponto de vista dos pacientes, há baixa escolaridade (que dificulta a comunicação com os médicos) e procura inadequada por serviços de pronto-socorro. Do ponto de vista dos profissionais, há aumento da burocracia; aumento da responsabilidade e diminuição da autonomia; assalariamento da classe médica, com consequências de baixa remuneração e baixa atratividade da APS; crença de que há "obstáculos culturais" em seus pacientes e percepção de que um "trabalho social" atrapalha a clínica médica. Os autores sugeriram educar os pacientes para uso correto dos serviços e promover a participação dos médicos na formulação das políticas de saúde.

Cardarelli e Chiapa (2007) destacaram três fatores que influenciam negativamente o encontro clínico: preconceito racial, maior incerteza clínica ao lidar com pacientes provenientes de minorias e crenças estereotipadas sobre 
as práticas cotidianas e de saúde desses grupos. Preconizaram que as equipes discutam sobre a questão étnica, para aumentar a consciência de possíveis preconceitos e estereótipos que podem, de forma mascarada, informar suas práticas. Para os autores, os profissionais podem conciliar o foco no paciente, a consciência sobre suas peculiaridades culturais e a prática da MBE.

DiMatteo, Murray e Williams (2009) investigaram o papel do gênero na comunicação entre pacientes e profissionais de diferentes origens étnicas e mostraram que as relações são mais favoráveis quando a díade é do mesmo gênero. Jones et al. (2008) pesquisaram as percepções de profissionais de saúde quanto às dificuldades de acesso aos serviços enfrentadas por pessoas com deficiência mental e verificaram que esses profissionais não se sentem preparados para se comunicar efetivamente com esses pacientes.

Os estudos que descrevemos até aqui têm em comum o fato de mostrarem que "visões estereotipadas" (sobre profissionais e pacientes) estão sempre presentes na organização geral dos sistemas de saúde e em cada encontro clínico. Interessa-nos considerar a construção social dessas "visōes" e sugeriremos, mais adiante, que a Psicologia Social pode contribuir para essa tarefa.

Outro conjunto de estudos enfatizou as tomadas de decisão que ocorrem na clínica. Kaner et al. (2007) deram importância especial à noção de tomada de decisão compartilhada ("shared decision making") entre profissional e paciente. Pesquisaram as ajudas computadorizadas para decisão compartilhada e verificaram que elas não reforçaram o compartilhamento de decisões entre médicos e pacientes, pois os médicos continuaram a dominar a fala no encontro clínico, utilizando discurso mais técnico-informativo, com poucas expressões sócio-afetivas.

Tracy, Dantas e Upshur (2003) enfocaram o processo de tomada de decisão, pesquisando a percepção dos médicos sobre MBE e a influência das preferências dos pacientes e da intuição do profissional. Os autores ressaltaram a insuficiência de tomar as evidências científicas como única base para prática, uma vez que os médicos mostraram acreditar em forte influência das preferências dos pacientes e de julgamentos clínicos intuitivos. Já Forrest et al. (2006) pesquisaram especificamente o comportamento de "encaminhamento para um especialista", que pode se constituir em ameaça ao papel central da APS nos sistemas de saúde. Mostraram que o encaminhamento é resultado de uma soma de fatores, dos quais 
destacamos uma "menor tolerância à incerteza por parte do médico" e admitiram a dificuldade de estabelecer critérios claros e definitivos para o encaminhamento.

As relações entre profissionais e pacientes são um aspecto central da APS. As dificuldades que podem advir desses encontros, e que foram citadas nos estudos acima, se colocam como preocupação para gestores e pesquisadores. Há iniciativas de educação continuada que apresentam o objetivo de melhorar as habilidades interpessoais dos profissionais. Cheraghi-Sohi e Bower (2008) realizaram uma revisão da literatura produzida a partir de ensaios clínicos randomizados que testaram efeitos de intervenções desse tipo. Apesar de algumas transformações positivas, os resultados foram considerados pelos autores como inconclusivos. Eles recomendaram que intervenções com esse objetivo tenham embasamento teórico mais forte, utilizando referências de outras disciplinas e combinando múltiplos métodos.

Os estudos cujo tema foi a relação entre profissionais e pacientes fizeram referência quase exclusivamente ao profissional de medicina. De forma geral, na literatura analisada, falar em APS parece ter o significado de falar do médico. Entretanto, três estudos enfocaram especificamente a ação do enfermeiro. De forma emblemática, o estudo teórico de Geden, Isaramalai e Taylor (2001) buscou reforçar a especificidade da atuação do enfermeiro, qual seja, a de promover o autocuidado junto ao paciente (para além de procedimentos biomédicos ou de prescrição). Já Randell et al. (2009) analisaram usos e atitudes de enfermeiros com relação a instrumentos para tomada de decisão, mostrando que os profissionais em geral preferem basear-se em sua experiência e sugerindo que esses instrumentos sejam reelaborados levando em conta especificidades concretas dos encontros clínicos (para além das chamadas "evidências"). Por fim, Lenz et al. (2004) demonstraram, em consonância com um número crescente de estudos, que a qualidade da APS dispensada por enfermeiros é equivalente àquela provida por médicos (entrevistando e examinando cerca de 400 pacientes).

\section{c) Avaliação de programas e intervenções}

Quatro artigos pesquisados centraram foco em iniciativas de intervenção na APS. Dois deles se apresentaram propriamente como "relatos de intervenção". O primeiro (HOKE et al., 2002) descreve um projeto de implementação de educação para a saúde em um serviço de APS, sugerindo profissionais específicos para esse fim. 
O segundo (SHARMA et al., 2001) partiu da necessidade de melhor preparar os profissionais da APS para lidar com os transtornos mentais mais comuns (destaca-se, de passagem, a pouca representatividade do tema "saúde mental" na amostra que analisamos). O programa descrito cuidava diretamente de pacientes com quadros mais graves e fornecia apoio para que as equipes de APS se ocupassem dos demais casos. O programa se mostrou satisfatório para médicos e pacientes. As equipes se aproximaram mais do tema "saúde mental" com diminuição dos estigmas, redução de encaminhamentos e redução de mais de $38 \%$ das internações.

Schenström, Rönnberg e Bodlund (2006), preocupados com a saúde mental dos próprios profissionais, avaliaram os resultados de um programa de treinamento cognitivo dirigido a vários profissionais e obtiveram mostras de que a intervenção diminuiu stress, aumentou qualidade de vida e melhorou o atendimento. Por fim, Mankuta et al. (1999) buscaram mensurar resultados de um projeto de gerenciamento da qualidade implantado em clínicas de APS e verificaram que houve melhora nos relatos médicos e no uso dos serviços complementares, mas que não houve alterações nas relações entre médicos e pacientes. Os médicos apresentaram baixos escores em habilidades psicossociais, prevenção e educação em saúde, mostrando que o ponto das "relações interpessoais" pode ser o mais sensível e urgente nas intervençôes.

\section{Discussão}

Coelho (2008) afirma que, ao longo de décadas dos séculos XIX e XX, os modelos de organização dos sistemas de saúde se preocuparam essencialmente com a distribuição de hospitais e médicos. Entretanto, diversos fatores sociais, políticos e econômicos alteraram esse quadro. $\mathrm{O}$ autor cita dados que mostraram o pronunciado aumento dos gastos públicos com saúde, no decorrer do século XX, em países ditos "desenvolvidos”. Esses gastos são correlatos de um grande aumento da procura por hospitais e ao desenvolvimento de tecnologias e equipamentos médicos mais sofisticados e mais caros. Ao mesmo tempo, estudos mostraram que o "estilo de vida" é a variável mais importante para a determinação dos níveis de saúde de uma população. Estudiosos criticaram a iatrogenia ligada aos hospitais e à crescente indústria de exames. Além disso, as últimas décadas do século XX foram caracterizadas por políticas 
neoliberais, que pressionaram pela diminuição dos gastos estatais com o setor

de saúde. Todos esses fatores são apontados pelo autor como determinantes da revalorização dos níveis de atenção primária à saúde, da prevenção de doenças e da promoção de estilos de vida saudáveis (COELHO, 2008).

As apostas na atenção primária são altas e crescentes. Segundo a Organização Mundial da Saúde (1978), ela deve ser a garantia de entrada no sistema e deve ser resolutiva para a grande maioria dos problemas de saúde da população. A análise histórica e crítica de modelos de atenção à saúde e da Declaração de Alma-Ata operada por Franco e Merhy (2007) evidenciam a pluralidade de determinações políticas, econômicas e sociais na implantação das práticas de saúde. Os autores afirmam que o discurso dessa Declaração mantém uma racionalidade de hierarquização dos procedimentos em saúde e não questiona a centralização na figura do médico. Não se aborda a dinâmica de interaçôes entre profissionais e usuários como elemento fundamental do trabalho na APS e não se considera a importância dos movimentos instituintes de novas relações humanas na produção de saúde. Para os autores, as práticas da APS, apesar de seu instrumental aparentemente mais simples, são extremamente complexas (sobretudo no nível das "tecnologias humanas"). Essas práticas devem integrar epidemiologia e clínica, fundando novas formas de relação entre profissionais e usuários, baseadas no vínculo e na autonomia.

Em consonância com essas apostas e com essa complexidade, a lista de recomendaçôes dirigidas à APS, nos estudos que analisamos, é heterogênea e muito extensa. Segundo os autores, é preciso que a prática clínica incorpore as evidências científicas, utilizando suportes impressos e eletrônicos e que, ao mesmo tempo, esteja focada no usuário e em suas possíveis especificidades culturais, de raça e de gênero; que os profissionais vejam os pacientes com uma perspectiva holística, que enfatizem a prevenção e a promoção de um estilo de vida saudável; que as decisões sobre o curso do tratamento sejam tomadas efetivamente em conjunto com o usuário; que os profissionais desenvolvam permanentemente seu saber clínico, que seu treinamento seja continuado e interdisciplinar, que funcionem de forma integrada e com foco em resultados e que discutam sobre questôes étnicas para evitar preconceitos.

Recomenda-se também que os serviços se aproximem da população atendida por meio de visitas domiciliares, que contem com o apoio de equipes consultivas 
e que ofereçam medicina alternativa complementar; que os serviços atendam a todos (universalidade), que sejam resolutivos e que previnam internaçóes, encaminhamentos e pedidos de exames desnecessários; que o sistema de saúde remunere melhor os profissionais e que dê maior autonomia a enfermeiros e auxiliares; que profissionais da área social integrem as equipes e que os profissionais participem da formulação das políticas de saúde e da gestão dos serviços.

Essas recomendações são condizentes com certas funções da APS, descritas por Campos et al. (2008). Os autores afirmam que cabe à APS visar à máxima resolutividade, estabelecer prioridades e praticar uma clínica ampliada. Entretanto, preconizam também que é preciso fomentar a corresponsabilidade dos usuários e sua postura ativa na produção da saúde, ponto que nos pareceu praticamente ausente da literatura internacional que analisamos. Os artigos não reservaram espaço para a participação popular. As recomendaçóes feitas pelos autores desses estudos mantêm plenamente o "lugar do especialista" que pensa, atua e decide pelo outro (usuário, comunidade). Essa valorização do especialismo se expressou também em certa fetichização do saber científico, percebida na ênfase dada às "evidências". Em uma palavra, os artigos não associaram a clínica à promoção de cidadania, autonomia e cogestão, essenciais para uma clínica ampliada (CAMPOS, 2003).

A articulação dos serviços em rede também está ausente dos artigos que analisamos. Alguns deles tomam como variável a existência de serviços complementares, mas não são feitas reflexões sobre a produção coletiva e concreta de uma rede de serviços, nem sobre a necessidade de intersetorialidade.

Tomemos agora os problemas identificados pelos artigos revisados: o contexto da APS é muito complexo e diverso, o que dificulta a implementação de diretrizes e protocolos (produzidos em quantidades excessivas); os profissionais podem estar desmotivados e com práticas cristalizadas, alguns acreditam que não conseguem mudar efetivamente o estilo de vida dos usuários; há profissionais que acreditam em obstáculos culturais intransponíveis para lidar com classes populares e com outras etnias. Há dificuldades em se aproximar das questôes de saúde mental. Falta tempo para centrar a atenção no usuário, em seu contexto e nas atividades preventivas (e essas ações são sub-remuneradas).

Há visões estereotipadas sobre a relação médico-paciente que preveem o fornecimento de uma solução imediata para a doença por parte do especialista; 
há médicos que não se sentem preparados para promover a prevenção e não há profissionais específicos para educação em saúde. Tomar decisões em conjunto com os usuários é tido como raro e difícil; médicos dominam a fala no encontro clínico e enfatizam interações técnicas em detrimento das sócio-afetivas. Faltam médicos generalistas, já que se trata de uma posição desvalorizada financeira e socialmente; a quantidade de usuários por profissional é sempre excessiva e pessoas com baixa renda, minorias étnicas e pessoas com deficiências têm acesso significativamente mais difícil à APS.

Seguindo a argumentação de Campos et al. (2008), pode-se afirmar que a APS brasileira enfrenta problemas semelhantes. Os autores enfatizam que os profissionais em geral não são formados para o atendimento comunitário e para a promoção de saúde. Em geral não contam com apoio técnico, não obtêm satisfação no trabalho, valorização, nem perspectiva de crescimento profissional. Os autores discutem especificamente o trabalho do médico: “[...] em todos os países do mundo, nenhuma estratégia para a APS funcionou sem alguma solução para o trabalho médico. A crítica à biomedicina, para alguns, transforma-se em combate aos médicos em geral" (CAMPOS et al., 2008, p. 149). Para os autores, o hipercriticismo ao modelo biomédico pode paradoxalmente o estar reforçando, na medida em que impede que o sistema público de saúde ofereça efetivamente atendimento de qualidade (o que valoriza o mercado dos serviços privados).

Sousa e Hamann (2009) identificam outros problemas relativos ao caso brasileiro. A Estratégia Saúde da Família (ESF) encontra sérias dificuldades para sua implementação em grandes cidades, incluindo disputas político-partidárias e violência urbana. Há incapacidade dos municípios em complementar a verba destinada pelo Governo Federal à APS, limitando a expansão da rede. Nenhuma solução consistente foi apresentada para o problema da capacitação permanente dos profissionais. Conclui-se que a implementação da ESF é uma agenda inerte e incompleta, por não ter sido capaz de "ampliar as condições para a superação efetiva do modelo biomédico hegemônico nas políticas públicas do setor saúde no Brasil" (SOUSA; HAMANN, 2009, p. 1334).

A literatura internacional que analisamos mostra que o que se pede da APS é muito e que os problemas são também diversos e difíceis. Percebemos que esses desafios são mundiais e que não foram totalmente equacionados. De muitas formas evidencia-se um descompasso entre a APS ideal e aquela 
realmente existente. Vimos que boa parte da literatura analisada considera que essa "APS ideal" deve levar em conta os modos de vida da população atendida. Visões e crenças compartilhadas entre profissionais, gestores e usuários têm papel crucial na organização do serviço, no encontro clínico e na determinação de sua eficácia.

Verificamos aqui uma contradição na literatura internacional analisada. Os autores afirmaram a importância de percepçōes, crenças, valores e relações interpessoais, mas não se utilizaram de instrumentos teóricos para compreendêlos (instrumentos que foram desenvolvidos pelas ciências humanas). Para pensar essa contradição, talvez seja conveniente diferenciar "abordagem" e "teoria". Os artigos utilizaram argumentos com feições de "abordagem" (diretrizes para uma prática, com função informativa), em detrimento do uso de "teoria" (visão de homem e de mundo, com função explicativa). Nesses artigos, é como se o campo da saúde tivesse que considerar o "humano", mas sem se aproximar das "ciências humanas". Percebe-se ausência de leitura crítica, histórica, social e psicossocial capaz de explicar crenças, representações e valores construídos por profissionais e usuários (sobre saúde, doença, sobre o que é ser profissional de saúde, o que é ser médico, usuário, etc.).

Para concluir, gostaríamos de sugerir que a psicologia social pode fornecer contribuiçôes importantes para ajudar a equacionar as demandas e os problemas associados à APS. A título de exemplo, gostaríamos de citar o trabalho de Apostolidis (2006), que parte da psicologia social da saúde como campo de estudos e de intervenções. A Teoria das Representaçōes Sociais, que embasa esse campo, permite examinar a construção de pensamentos e práticas sobre saúde e doença e é um quadro teórico integrador que propõe analisar simultaneamente dimensões sociais e psicológicas (conhecimentos, crenças, afetos e práticas).

As práticas de saúde são práticas sociais, imersas em jogos complexos de significação, afetividade e valor; jogos associados às identidades sociais, às relações de gênero, aos estereótipos, preconceitos e representaçôes. Como praticar a promoção de saúde, a prevenção e a clínica na APS, como promover participação popular, corresponsabilização e autonomia, como ajudar a população a reverter a lógica curativista, sem considerar esses sistemas de valores e de representaçôes sociais que constroem seu mundo cotidiano? 
A Atenção Primária à Saúde ocupa um lugar central e estratégico nos sistemas nacionais de saúde. Nos artigos revisados, encontram-se numerosas demandas e recomendações para a organização dos serviços e para as práticas dos profissionais. Essas demandas e recomendações são consoantes com parâmetros estabelecidos em âmbito internacional: a APS deve ser a porta de entrada e o principal ponto de contato com o sistema de saúde, deve ser resolutiva e deve basear-se no vínculo e no cuidado longitudinal, transformando concretamente padrões e estilos de vida da população atendida. Entretanto, a análise da literatura internacional revelou diversos problemas ainda não solucionados, detectados também na realidade brasileira, como dificuldades em construir relações duradouras com os usuários, dificuldades em abordar questões de saúde mental, dificuldades em consolidar uma clínica preventiva e, como decorrência, dificuldades em transformar estilos de vida dos usuários.

Vimos que a literatura analisada é centrada hegemonicamente no profissional de medicina e que mantêm plenamente o "lugar de especialista" para os profissionais, ou seja, o lugar daquele que "decide e que faz pelo outro". Em nossa discussão, destacamos que uma inflexão nesse ponto, a construção do usuário como participante ativo dos processos de saúde, parece ser fundamental para uma consecução mais plena dos objetivos da APS. Sinalizamos que os trabalhos revisados reconheceram a importância de percepçóes, crenças e valores adotados por profissionais e por usuários. Reconheceram, portanto, a importância de "fatores humanos", mas se mostraram distantes das ciências humanas. Para finalizar, sugerimos que a clínica e a pesquisa em APS considerem sistematicamente as representações e as práticas sociais dos profissionais e das populações atendidas, ou seja, as formas pelas quais eles constroem sua realidade social e sanitária.

\section{Referências}

APOSTOLIDIS, T. Representations sociales et triangulation: une application en psychologie sociale de la santé. Psicologia teoria e pesquisa, v. 22, n. 2, p. 211-226, 2006.

BAPTISTA, T.W.F.; FAUSTO, M.C.R.; CUNHA, M.S. Análise da produção bibliográfica sobre atenção primária à saúde no Brasil em quatro periódicos selecionados. Physis: revista de saúde coletiva, v. 19, n. 14, p. 1007-1028, 2009. 
BEAULIEU, M.D. et al. When is knowledge ripe for primary care? An exploratory study on the meaning of evidence. Evaluation \& the health professions, v. 31, n. 1, p. 22-42, 2008. BIBLIOTECA VIRTUAL EM SAÚDE. Portal BioMed Central sobre acesso aberto e países em desenvolvimento. 2007. [acessado 2009 Set 19]. Disponível em: http://cvirtualccs.bvsalud.org/ tikiread_article.php?articleId=183\& highlight $=$ rsp.

CAMPOS, G.W.S. et al. Reflexōes sobre a atenção básica e a estratégia de saúde da família. In: CAMPOS, G.W.S.; GUERRERO, A.V.P. (Org.). Manual de práticas de atenção básica: saúde ampliada e compartilhada. São Paulo: Hucitec, 2008. p. 132-153.

CAMPOS, G.W.S. Saúde paideia. São Paulo: Hucitec, 2003.

CARDARELLI, R.; CHIAPA, A.L. Educating primary care clinicians about health disparities. Osteopathic Medicine and Primary Care, v. 1, n. 5, 2007.

CHAUDRY, R.V. et al. Caring for patients under Medicaid Mandatory Managed Care: perspectives of primary care physicians. Qualitative Health Research, v. 13, n. 1, p. 37-56, 2003. CHERAGHI-SOHI, S.; BOWER, P. Can the feedback of patient assessments, brief training, or their combination, improve the interpersonal skills of primary care physicians? A systematic review. BMC Health Services Research, v. 8, n. 179, 2008.

COELHO, I.B. Formas de pensar e organizar o sistema de saúde: os modelos assistenciais em saúde. In: CAMPOS, G.W.S.; GUERRERO, A.V.P. (Org.). Manual de práticas de atenção básica: saúde ampliada e compartilhada. São Paulo: Hucitec, 2008. p. 96-131.

DIMATTEO, M.R.; MURRAY, C.B.; WILLIAMS, S.L. Gender disparities in physicianpatient communication among african american patients in primary care. Journal of Black Psychology, v. 35, n. 2, p. 204-227, 2009.

DOBSCHA, S.K. et al. Primary care provider preferences for working with a collaborative support team. Implementation Science, v. 2, n. 16, 2007.

FEIFER, C. et al. The logic behind a multimethod intervention to improve adherence to clinical practice guidelines in a nationwide network of primary care practices. Evaluation \& the health professions, v. 29, n. 1, p. 65-88, 2006.

FORREST, C.B. et al. Primary care physician specialty referral decision making: patient, physician, and health care system determinants. Medical decision making, v. 26, p. 76-85, 2006.

FRANCO, T.B.; MERHY, E.E. Programa de saúde da família (PSF): contradições de um programa destinado à mudança do modelo tecnoassistencial. In: MERHY E.E. et al. O trabalho em saúde: olhando e experienciando o SUS no cotidiano. São Paulo: Hucitec, 2007. p. 55-124.

FREEBORN, D.K.; HOOKER, R.S.; POPE, C.R. Satisfaction and well-being of primary care providers in managed care. Evaluation \& the health professions, v. 25, n. 2, p. 239-254, 2002. 
GEDEN E.A.; ISARAMALAI, S.; TAYLOR S.G. Self-care deficit nursing theory and the nurse practitioner's practice in primary care settings. Nursing Science Quarterly, v. 14, n. 1, p. 30-33, 2001.

GENEAU, R. et al. Understanding the work of general practitioners: a social science perspective on the context of medical decision making in primary care. BMC Family Practice, v. 9, n. 12, 2008.

GIOVANELLA, L. A atenção primária à saúde nos países da União Europeia: configurações e reformas organizacionais na década de 1990. Cadernos de saúde pública, v. 22, n. 5, p. 951$963,2006$.

GRANDES, G. et al. Is integration of healthy lifestyle promotion into primary care feasible? Discussion and consensus sessions between clinicians and researchers. BMC Health Services Research, v. 8, n. 213, 2008.

GUERRA, C.E. et al. Effect of guidelines on primary care physician use of PSA screening: results from the community tracking study physician survey. Medical decision making, v. 28, p. 681-689, 2008.

GUNTHER, H. Pesquisa qualitativa versus pesquisa quantitativa: esta é a questão? Psicologia teoria e pesquisa, v. 22, n. 2, p. 201-209, 2006.

HOKE, M.M. et al. The clinic HELP initiative: promoting health education in primary care clinics. Health Promotion Practice, v. 3, n. 4, p. 477-484, 2002.

HSIAO, C.; BOULT, C. Effects of quality on outcomes in primary care: a review of the literature. American Journal of Medical Quality, v. 23, n. 4, p. 302-310, 2008.

HUNTER, D.J.W. et al. Family physician views about primary care reform in Ontario: a postal questionnaire. BMC Family Practice, v. 5, n. 2, 2004.

JONES, M.C. et al. Inclusion in primary care for people with intellectual disabilities. Gaining the perspective of service user and supporting social care staff. Journal of Intellectual Disabilities, v. 12, n. 2, p. 93-109, 2008.

KANER, E. et al. Medical communication and technology: a video-based process study of the use of decision aids in primary care consultations. BMC Medical Informatics and Decision Making, v. 7, n. 2, 2007.

LENZ, E.R. et al. Primary care outcomes in patients treated by nurse practitioners or physicians: two-year follow-up. Medical Care Research and Review, v. 61, n. 3, p. 332-351, 2004.

LEONARDO, M.E.; CHECCO, B.J.; COGGINS, G.A. Home visits in rural Nicaragua: a transcultural primary care experience. Home Health Care Management Practice, v. 13, n. 3, p. 197-206, 2001.

LOPES, A.A. Medicina baseada em evidências: a arte de aplicar o conhecimento científico na prática clínica. Revista da Associação Médica Brasileira, v. 46, n. 3, p. 285-288, 2000. 
MANKUTA, D. et al. A quality management project in israeli navy primary care clinics. American Journal of Medical Quality, v. 14, n. 5, p. 211-215, 1999.

MARTIN-FERNANDEZ J. et al. Professional quality of life and organizational changes: a five-year observational study in Primary Care. BMC Health Services Research, v. 7, n. 101, 2007. MAY, C.R. et al. Process evaluation for complex interventions in primary care: understanding trials using the normalization process model. BMC Family Practice, v. 8, n. 42, 2007.

MIRAND, A.L. et al. Explaining the de-prioritization of primary prevention: physicians' perceptions of their role in the delivery of primary care. BMC Public Health, v. 3, n. 15, 2003. NEMETH, L.S. et al. Implementing change in primary care practices using electronic medical records: a conceptual framework. Implementation Science, v. 3, n. 3, 2008.

ORGANIZAÇĀO MUNDIAL DA SAÚDE. Declaração de Alma-Ata. Genebra: OMS, 1978. POLLAK, K.I. et al. Estimated time spent on preventive services by primary care physicians. BMC Health Services Research, v. 8, n. 245, 2008.

RANDELL, R. et al. Supporting nurse decision making in primary care: exploring use of and attitude to decision tools. Health Informatics Journal, v. 15, n. 1, p. 5-16, 2009.

RINKE, L.T.; HOLT, S.W. Primary care in the home: the time is now! Home Health Care Management Practice, v. 12, n. 3, p. 1-9, 2000.

SAYD, J.D.; MOREIRA, M.C.N. Medicina baseada em evidências - ceticismo terapêutico, recorrência e história. Physis: revista de saúde coletiva, v. 10, n. 1, p. 11-38, 2000.

SCHENSTRÖM, A.; RÖNNBERG, S.; BODLUND, O. Mindfulness-based cognitive attitude training for primary care staff: a pilot study. Complementary Health Practice Review, v. 11, n. 3, p. 144-152, 2006.

SHARMA, V.K. et al. Developing mental health services in a primary care setting: Liverpool primary care mental health project. International Journal of Social Psychiatry, v. 47, n. 4, p. 16-29, 2001.

SOUSA, M.F.; HAMANN, E.M. Programa Saúde da Família no Brasil: uma agenda incompleta? Ciência e saúde coletiva, v. 14, supl. 1, p. 1325-1335, 2009.

STRANDBERG, E.L. et al. The perceived meaning of a (w)holistic view among general practitioners and district nurses in Swedish primary care: a qualitative study. BMC Family Practice, v. 8, n. 8, 2007.

SUNDBERG, T. et al. Towards a model for integrative medicine in Swedish primary care. BMC Health Services Research, v. 7, n. 107, 2007.

TRACY, C.S. et al. The nexus of evidence, context, and patient preferences in primary care: postal survey of Canadian family physicians. BMC Family Practice, v. 4, n. 13, 2003.

TRACY, C.S.; DANTAS, G.C.; UPSHUR, R.E.G. Evidence-based medicine in primary care: qualitative study of family physicians. BMC Family Practice, v. 4, n. 6, 2003. 
Primary health care: guidelines, challenges and recommendations. A review of international literature

We present a review of international literature, aiming at identifying and analyzing the diversity of themes associated to Primary Health Care. We focused on the characteristics of the work of health care personnel, its guidelines and its political and clinical challenges. We analyzed the abstracts of 105 articles published between 1999 and 2009. Then, we integrally read and analyzed 34 articles, creating thematic categories. We compared data to Brazilian literature on the subject. The articles focus on physician's work and emphasize the notion of evidence-based medicine. They mainly approach the implementation of guidelines to primary health care and the professional-patient relations. They acknowledge the complexity of Primary Care and the importance of patient-centered care. However, they do not mention integrated actions with other public policies. The authors do not radically overthrow the cure-centered specialism and they do not write about fostering patients' participation, autonomy and empowerment. They acknowledge the need to approach "human" issues at the levels of guidelines implementation, clinical practices etc. Nevertheless, in general, these authors are far from the social sciences.

Key words: primary health care, health personnel, health planning guidelines, evidence-based medicine. 